\title{
8 Berücksichtigung der Regulierungsmöglichkeiten bei der Begründung einer Reaktionspflicht
}

Ausgangspunkt der Überlegungen zur Begründung einer Reaktionspflicht war, dass als „Zünglein an der Waage“ - entscheidend ist, ob den Ländern Optionen zur Verfügung stehen, den Ordnungsrahmen jenseits einer symbolhaften oder kontraproduktiven Gesetzgebung zu modernisieren.

Auf Basis des soeben Ausgeführten bieten sich die folgenden Möglichkeiten an:

- Die gesetzliche Verankerung einer Suchmaschinenneutralität, angelehnt an wettbewerbsrechtliche Beurteilungsmaßstäbe und in Kombination mit einer Anreizregulierung für die Anbieter inhaltsgeprägter Telemedien.

- Die Etablierung eines öffentlich geförderten Suchindex als Ausgangsbasis für privatwirtschaftliche Entwicklungen.

- Die Verankerung von Transparenzpflichten, um die Funktionserwartung der Suchmaschinenutzer zu schützen, sowie als Hilfsmittel zur Beurteilung der Suchmaschinenneutralität.

- Die Etablierung einer Begleitforschung als Ausgangsbasis für zukünftige Reformen der Medienordnung.

- Die Kooperation mit Suchanbietern im Wege der Anreizregulierung über die Erweiterung des Medienprivilegs auf Suchmaschinen.

Mit Blick auf diese Möglichkeiten und die proaktive Natur der positiven Rundfunkordnung erscheint daher eine Reaktionspflicht des Gesetzgebers gegenwärtig begründet. 